\title{
TITLE:
}

\section{Consistency Proof via Pointwise Induction}

$\operatorname{AUTHOR}(\mathrm{S}):$

Arai, Toshiyasu

CITATION:

Arai, Toshiyasu. Consistency Proof via Pointwise Induction. 数理解析研 究所講究録 1997, 976: 125-134

ISSUE DATE:

1997-02

URL:

http://hdl.handle.net/2433/60800

RIGHT: 


\title{
Consistency Proof via Pointwise Induction
}

\author{
Toshiyasu Arai (新井敏康) \\ Faculty of Integrated Arts and Sciences \\ Hiroshima University
}

\begin{abstract}
We show that the consistency of the first order arithmetic $P A$ follows from the pointwise induction up to the Howard ordinal. Our proof differs from U. Schmerl [S]: We do not need Girard's Hierarchy Comparison Theorem. A modification on ordinal assignment to proofs by Gentzen and Takeuti [T] is made so that one step reduction on proofs exactly corresponds to the stepping down $\alpha \mapsto \alpha[1]$ in ordinals. Also a generalization to theories $I D_{q}$ of finitely iterated inductive definitions is proved.
\end{abstract}

We show that the consistency of the first order arithmetic $P A$ follows from the pointwise induction up to the Howard ordinal. Our proof differs from U. Schmerl [S]: We do not need Girard's Hierarchy Comparison Theorem.

Let $P$ be a proof of the empty sequent in $P A$ or the second order arithmetic $\Pi_{1}^{1}-C A_{0}$. For such a proof $P$ let $o(P)$ denote the ordinal assigned to $P$ and $r(P)$ a reduct of $P$ defined by Gentzen and Takeuti $[\mathrm{T}] . r(P)$ is again a proof of the empty sequent and $o(r(P))<o(P)$. Then the reduction $r: P \mapsto r(P)$ is close to but does not fit perfectly the stepping down $\alpha \mapsto \alpha[n]$ defined by Buchholz [B2]. ${ }^{1}$ We need to tune these functions $O$ and $r$ to stepping down in order to have $o(r(P))=o(P)[n]$ for an $n$. For this purpose we introduce two inference rules: the padding rule and the height rule. In both rules the lowersequent is identical with the uppersequent. Let $S_{u}\left[S_{l}\right]$ denote the uppersequent [the lowersequent], resp. Also let $h(S)$ denote the height of a sequent (in a proof $P$ ).

$$
\frac{\Gamma}{\Gamma}(p a d)_{b}
$$

with $o\left(S_{l}\right)=o\left(S_{u}\right)+b$.

$$
\frac{\Gamma}{\Gamma}(h g t)
$$

with $h\left(S_{u}\right)=h\left(S_{l}\right)+1$ and $o\left(S_{l}\right)=D_{1} o\left(S_{u}\right)$. $D_{1} a$ denotes an ordinal term defined in [B2].

Using these rules we can unwind gaps between $D$ and $r(D)$ so that

$o(r(P))=o(P)[1]$ holds.

\section{Fundamental sequences}

The following is the fundamental sequences given in [A] and a slight variant in Buchhloz [B2]. Let $q$ be a natural number.

Definition 1 (Buchholz [B2]) The term structure $(T(q), \cdot[\cdot])$

1. Inductive definition of the sets $P T(q)$ and $T(q)$

$$
\begin{aligned}
& \text { (T0) } P T(q) \subseteq T(q) \\
& \text { (T1) } 0 \in T(q) \\
& \text { (T2) } a \in T(q) \& 0 \leq u \leq q \Rightarrow D_{u} a \in P T(q) \\
& \text { (T3) } a_{0}, \ldots, a_{k} \in P T(q)(k>0) \Rightarrow\left(a_{0}, \ldots, a_{k}\right) \in T(q)
\end{aligned}
$$

2. For $a_{0}, \ldots, a_{k} \in P T(q)$ and $k \in\{-1,0\}$, we set

$$
\left(a_{0}, \ldots, a_{k}\right)= \begin{cases}0 & k=-1 \\ a_{0} & \text { otherwise }\end{cases}
$$

\footnotetext{
${ }^{1}$ This observation is also stated by M. Hamano and M. Okada [H-O].
} 
3. $a+0=0+a=a ;\left(a_{0}, \ldots, a_{k}\right)+\left(b_{0}, \ldots, b_{m}\right)=\left(a_{0}, \ldots, a_{k}, b_{0}, \ldots, b_{m}\right) ; a \cdot 0=0 ; a \cdot(n+1)=a \cdot n+a$

4. $\omega=d_{d f}\{0,1,1+1, \ldots\} \subset T(q)$ with $1=D_{0} 0$

5. For $u \leq q$, $T_{u}(q)=\left\{\left(D_{u_{0}} a_{0}, \ldots, D_{u_{k}} a_{k}\right): k \geq-1, a_{0}, \ldots, a_{k} \in T(q), u_{0}, \ldots, u_{k} \leq u\right\}$

6. $\operatorname{dom}(a)$ and $a[z]$ for $a \in T(q)$ and $z \in \operatorname{dom}(a)$

([ ].1) $\operatorname{dom}(0)=\emptyset$

([ ].2) $\operatorname{dom}(1)=\{0\} ; 1[0]=0$

([ ].3) $\operatorname{dom}\left(D_{u+1} 0\right)=T_{u}(q) ;\left(D_{u+1} 0\right)[z]=z$

([ ].4) Let $a=D_{v} b$ with $b \neq 0$.

(a) If $b=b_{0}+1$, then $\operatorname{dom}(a)=\omega ; a[z]=\left(D_{v} b_{0}\right) \cdot(n+1)$

(b) If $\operatorname{dom}(b) \in\{\omega\} \cup\left\{T_{u}(q): u<v\right\}$, then $\operatorname{dom}(a)=\operatorname{dom}(b) ; a[z]=D_{v} b[z]$

(c) If $\operatorname{dom}(b) \in\left\{T_{u}(q): u \geq v\right\}$, then $\operatorname{dom}(a)=\omega ; a[n]=D_{v} b\left[b_{n}\right]$ with $b_{0}=1, b_{m+1}=D_{u} b\left[b_{m}\right]$

([ ].5) $a=\left(a_{0}, \ldots, a_{k}\right) k>0: \operatorname{dom}(a)=\operatorname{dom}\left(a_{k}\right) ; a[z]=\left(a_{0}, \ldots, a_{k-1}\right)+a_{k}[z]$

Let $O T(q) \subset T(q)$ denote the set of ordinal terms in [B1]. For example $O T(1)$ corresponds to the Howard ordinal $\psi_{0} \varepsilon_{\Omega+1}$. In [B1] Buchholz shows that the proof theoretic ordinal of the theory $I D_{q}$ for $q$-fold iterated inductive definitions is given by the ordinal $\psi_{0} \varepsilon_{\Omega_{q}+1}$, i.e., the order type of $O T_{0}(q)$.

Proposition 1 (Buchholz [B1])

$$
a, z \in O T(q) \& z \in \operatorname{dom}(a) \Rightarrow a[z] \in O T(q)
$$

\section{Coventions.}

1. $\Omega_{q}=D_{d f} D_{q} 0$

2. $0[n]=0 ;(a+1)[n]=a$ for $n \in \omega$

3. $a[n]^{0}=a ; a[n]^{m+1}=\left(a[n]^{m}\right)[n]$

4. $D_{u}^{0} a=a ; D_{u}^{k+1} a=D_{u}\left(D_{u}^{k} a\right)$

ERA denotes the Elementary Recursive Arithmetic.

Let $(P I)_{q}$ denote the following inference rule:

$$
\frac{A(0, p) \quad \alpha \neq 0 \wedge A(\alpha[1], r(p)) \supset A(\alpha, p)}{A(\alpha, p)}(P I)_{q}
$$

where $\alpha$ denotes a variable ranging over $O T(q)$, and $A[r]$ is an elementary recursive relation $\in \mathcal{E}_{*}^{3}\left[\right.$ function $\left.\in \mathcal{E}^{3}\right]$, resp.

For a theory $T$ let $C o n^{(n)}(T)$ denote the iterated consistency of $T$ :

$$
\operatorname{Con}^{(0)}(T) \Leftrightarrow \forall x(0=0) ; \operatorname{Con}^{(n+1)}(T) \Leftrightarrow \operatorname{Con}\left(T+\operatorname{Con}^{(n)}(T)\right)
$$

Now our theorems are stated as follows:

Theorem 1 For each natural number $q$,

1. Over ERA, $\left\{\operatorname{Con}^{(n)}\left(I D_{q}\right): n<\omega\right\}$ is equivalent to $(P I)_{q+1}$.

2. Over $E R A$, the 1-consistency $R F N_{\Sigma_{1}}\left(I D_{q}\right)$ of $I D_{q}$ is equivalent to $\forall n \exists m\left\{\left(D_{0} D_{q+1}^{n}\left(\Omega_{q+1} \cdot n\right)\right)[1]^{m}=0\right\}$.

For provably total recursive functions we have, e.g.,

Proposition 2 For each provably total recursive function $f$ in $P A$, there exist $k$ and $d$ such that $\forall n[f(n) \leq$ $\left.d \cdot \mu m\left\{\left(D_{0} D_{1}^{k}\left(\Omega_{1}(k+n)\right)\right)[1]^{m}=0\right\}\right]$.

This is seen from a slight modification of the proof of the theorem and so we omit a proof.

Remark. U. Schmerl $[\mathrm{S}]$ gives a proof of a variant of the Theorem $1.1(q=0)$, i.e., for $P A$ via Girard's Hierarchy Comparison Theorem. In [S] the base theory (for us $E R A$ ) contains the fast growing functions $F_{\alpha}\left(\alpha<\varepsilon_{0}\right)$ and/or the slow growing functions $G_{\alpha}\left(\alpha<\psi_{0} \varepsilon_{\Omega+1}\right)$ and their defing equations. Hence it seems that Schmerl's result is incomparable to ours. 


\section{Proof of Theorem}

Fix a natural number $q$. We prove the Theorem 1.1. The Theorem 1.2 is proved similarly.

First consider the easy half: The rule $(P I)_{q+1}$ is a derived rule in $E R A+\left\{\operatorname{Con}^{(n)}\left(I D_{q}\right): n<\omega\right\}$.

Let $\operatorname{prov}_{T}$ denote a standard proof predicate for a theory $T$ and " $B$ " the gödel number of an expression $B$. This follows from the following fact which is shown in $[\mathrm{A}]$ :

Proposition 3 For some elementary recursive function $f$ we have

$$
E R A \vdash \forall a \in T_{0}(q+1)\left\{\operatorname{prov}_{I D_{q}}\left(f(a), " \forall n \exists m\left(\dot{a}[n]^{m}=0\right) "\right)\right\}
$$

Next consider the other half. Let $\forall x B(x)$ be a $\Pi_{1}^{0}$ sentence. $I D_{q}+\forall x B(x)$ denote the theory obtained from $I D_{q}$ by adding extra axioms $B(t)$ for an arbitrary term $t$. It suffices to show, in $E R A+(P I)_{q+1}$, Con $\left(I D_{q}+\forall x B(x)\right)$ under the assumption ' $\forall x B(x)$ is true'. Our proof is an adaption from Gentzen's and Takeuti's reduction in $[\mathrm{T}]$.

First $I D_{q}$ is embedded in a first order theory $N I D_{q+1}$. In the latter theory the universe $\omega$ of $I D_{q}$ is replaced by a constant $N$ and this constant is treated as if it were a $\Pi_{1}^{1}$ formula. Then as in [T] the inference rules for the constant $N$ are analysed by using a substitution rule. Also as mentioned above we introduce two new rules, the padding rule and the height rule to unwind gaps in Gentzen-Takeuti reduction. Now details follow.

The language $L$ of $I D_{q}$ consists of

1. function constants 0 and the successor ',

2. arithmetic predicate constants are lower elementary recursive relations $R \in \mathcal{L}_{*}^{2}$ and their negations $\neg R$,

3. the least fixed points $\left\{P_{u}\right\}_{1 \leq u \leq q}$ for a fixed positive operator form $\mathcal{A}\left(X^{+}, Y, n\right)$ and

4. logical symbols $\wedge, \vee, \forall, \exists$.

The negation $\neg A$ of a formula $A$ is defined by using de Morgan's law and the elimination of double negations. A prime formula $R\left(t_{1}, \ldots, t_{n}\right)$ or its negation $\neg R\left(t_{1}, \ldots, t_{n}\right)$ with an arithmetic predicate $R$ is an a.p.f.(arithmetic prime formula).

The axioms in $I D_{q}$ are axioms for function and arithmetic predicate constants, the induction axiom $(I A)$ and axioms (P.1), (P.2) of the least fixed points $\left\{P_{u}\right\}_{1 \leq u \leq q}$ for arbitrary formula $F$ :

(IA) $F(0) \wedge \forall x\left(F(x) \supset F\left(x^{\prime}\right)\right) \supset \forall x F(x)$

$(P .1) \mathcal{A}_{u}\left(P_{u}\right) \subseteq P_{u}$

$(P .2) \mathcal{A}_{u}(F) \subseteq F \supset P_{u} \subseteq F$

where $\mathcal{A}_{u}(X)=\left\{n: \mathcal{A}\left(X, \sum_{1 \leq v<u} P_{v}, n\right)\right\}$.

The language $L_{N}$ of $N I D_{q+1}$ consists of $L \cup\{N\} \cup\left\{X_{i}: i<\omega\right\}$ with a unary predicate constant $N$ and a list of unary predicates $X_{i}$. These unary predicates are denoted $X, Y$, etc. We sometimes write $P_{0}$ for the constant $N$. For a predicate constant $H \in\left\{P_{u}: u \leq q\right\} \cup\left\{X_{i}: i<\omega\right\}$, we write $t \in H$ for $H(t)$ and $t \notin H$ for $\neg H(t)$. A formula is said to be an $E$ formula if it is either an a.p.f. or a formula in one of the following shapes; $A \vee B, \exists x A$ or $t \notin H$ with $H \in\left\{P_{u}: u \leq q\right\} \cup\left\{X_{i}: i<\omega\right\}$. A formula is an $A$ formula if its negation is an $E$ formula. For a formula $A$ in $L$ let $A^{N}$ denote the result of restricting all quantifiers in $A$ to $N$. For each $u \leq q$ let $\mathcal{N}_{u}(X, t)$ denote the formula:

$$
\begin{gathered}
\mathcal{N}_{0}(X, t) \equiv 0 \in X \wedge \forall x\left(x \in X \supset x^{\prime} \in X\right) \supset t \in X ; \\
\mathcal{N}_{u}(X, t) \equiv \mathcal{A}_{u}^{N}(X) \subseteq X \supset t \in X(u \neq 0)
\end{gathered}
$$

$N I D_{q+1}$ is fomulated in Tait's calculus, i.e., one sided sequent calculus. Finite sets of formulae is called a sequent. Sequents are denoted by $\Gamma, \Delta$, etc.

Axioms in $N I D_{q+1}$ are:

logical axiom $\Gamma, \neg A, A$

where $A$ is either an a.p.f. or a formula of the shape $t \in X$.

arithmetic axiom $1 . \Gamma, \Delta_{R}$

where $\Delta_{R}$ consists of a.p.f'.s and corresponds to the definition of a lower elementary relation $R$.

2. $\Gamma, A$ for a true closed a.p.f. $A$. 
3. $\Gamma, \Delta_{0}$

where there exists a sequent $\Delta_{1}$ so that $\Delta=\Delta_{0} \cup \Delta_{1}$ is an instance of a defining axiom for $R$ in 1 and $\Delta_{1}$ consists solely of false closed a.p.f.'s.

Inference rules in $N I D_{q+1}$ are:

$(\wedge),(\vee),(\forall),(\exists),(c u t),(w e a k)$ and $\left(P_{u}\right),\left(\neg P_{u}\right)$ for $(u \leq q)$.

1. $(\wedge),(\vee),(\forall),(\exists)$ : In these rules the principal formula is contained in the uppersequent. For example

$$
\frac{\Gamma, \exists x A(x), A(t)}{\Gamma, \exists x A(x)}(\exists)
$$

2. In the rule (cut)

$$
\frac{\Gamma, \neg A \quad A, \Delta}{\Gamma, \Delta}(c u t)
$$

the cut formula $A$ is an $E$ formula.

3. (weak) is the weakening:

$$
\frac{\Gamma}{\Delta}(w e a k)
$$

with $\Gamma \subseteq \Delta$.

4. $\left(P_{u}\right)$ :

$$
\frac{\Gamma, t \in P_{u}, \mathcal{N}_{u}(X, t)}{\Gamma, t \in P_{u}}\left(P_{u}\right)
$$

where $X$ is the eigenvariavle, i.e., does not occur in the lowersequent.

5. $\left(\neg P_{u}\right)$ :

$$
\frac{\Gamma, t \notin P_{u}, \neg \mathcal{N}_{u}(F, t)}{\Gamma, t \notin P_{u}}\left(\neg P_{u}\right)
$$

for an arbitrary formula $F$ in the language $L_{N}$.

Lemma 1 For any sentence $A$ in $L$,

$$
I D_{q} \vdash A \Rightarrow N I D_{q+1} \vdash A^{N}
$$

Proof. It suffices to show that the following sequents are provable in $N I D_{q+1}$ :

$t \notin P_{u}, t \in P_{u}$ : This is proved by induction on $u \leq q$. By $\operatorname{IH}\left(=\operatorname{Induction}\right.$ Hypothesis) we have $\neg \mathcal{N}_{u}(X, t), \mathcal{N}_{u}(X, t)$. Rules $\left(P_{u}\right)$ and $\left(\neg P_{u}\right)$ yields $t \notin P_{u}, t \in P_{u}$.

$(I A)^{N}$ : For a given formula $F(x)$ assume $a \in N, F(0)$ and $\forall x \in N\left(F(x) \supset F\left(x^{\prime}\right)\right)$. We have to show $F(a)$. Let $G(x)$ denote the fomula $x \in N \wedge F(x)$. Then we see $\forall x\left(G(x) \supset G\left(x^{\prime}\right)\right)$ from $x \in N \supset x^{\prime} \in N$. The latter follows from the rules $(\neg N)=\left(\neg P_{0}\right)$ and $(N)$. Also we have $G(0)$. On the other hand we have $\mathcal{N}_{0}(G, a)$ by the rule $(\neg N)$ and $a \in N$. Thus we get $G(a)$ and hence $F(a)$.

$(P .1)^{N}$ : Assume $\mathcal{A}_{u}^{N}\left(P_{u}, a\right)$. We have to show $a \in P_{u}$. By the rule $\left(P_{u}\right)$ it suffices to show $\mathcal{N}_{u}(X, a)$. Assume $\mathcal{A}_{u}^{N}(X) \subseteq X$. We show $a \in X$.

Claim. $P_{u} \subseteq X$

Proof of the Claim. Assume $x \in P_{u}$. By the rule $\left(\neg P_{u}\right)$ we have $\mathcal{N}_{u}(X, x)$. The assumption $\mathcal{A}_{u}^{N}(X) \subseteq$ $X$ yields $x \in X$.

From this Claim and the positivity of $X$ in $\mathcal{A}$ we see $\mathcal{A}_{u}^{N}(X, a)$. Thus again by the assumption $\mathcal{A}_{u}^{N}(X) \subseteq X$ we conclude $a \in X$.

$(P .2)^{N}$ : For a given formula $F$ assume $\mathcal{A}_{u}^{N}(F) \subseteq F$ and $a \in N \cap P_{u}$. We show $F(a)$. This follows from the rule $\left(\neg P_{u}\right)$. 
1. $|A|=0$ for a prime formula $A$. Specifically $|(\neg) H(t)|=0$ for any predicate $H$.

2. $|Q x A|=|F|+1$ for $Q \in\{\forall, \exists\}$

3. $\left|A_{0} \circ A_{1}\right|=\max \left\{\left|A_{i}\right|+1: i=0,1\right\}$ for $\circ \in\{\wedge, \vee\}$.

A formula $A \in P_{o s}$ iff 1 . if a predicate $P_{v}$ occurs positively in $A$, then $v \leq u$, and 2. if a predicate $P_{v}$ occurs negatively in $A$, then $v<u$.

Observe that $\mathcal{N}_{u}(X, t) \in \operatorname{Pos}_{u}$ and $\neg P_{u}(t) \notin P o s_{v} \& \operatorname{Pos}_{v} \subseteq P_{o s}$ for $v \leq u$.

Let $\forall x B(x)$ denote a fixed true $\Pi_{1}^{0}$ sentence with an a.p.f. $B$. The system $N I D_{q+1}+\forall x B(x)$ is obtained from $N I D_{q+1}$ by adding the axiom

$$
\text { (B) } \Gamma, B(t)
$$

for an arbitrary term $t$ and three inference rules; the padding rule $(p a d)_{b}(b \in O T(q+1))$, the height rule (hgt) mentioned in the introduction and the substitution rule $(s u b)_{u}(u \leq q)$.

$$
\frac{\Gamma(X)}{\Gamma(F)}(s u b)_{u}
$$

where 1. $\Gamma(X) \subseteq \operatorname{Pos}_{u}, 2$. $X$ is the eigenvariable, i.e., does not occur the lowersequent, 3. $F$ is an arbitrary formula in $L_{N}$ and 4 . $\Gamma(F)$ denotes the result of substituting $F$ for $X$ in $\Gamma(X)$.

Let $P$ be a proof (in $N I D_{q+1}+\forall x B(x)$ ) and $\Gamma$ a sequent in $P$. We define the height $h(\Gamma)=h(\Gamma ; P)$ of $\Gamma$ in $P$ as follows:

1. $h(\Gamma)=0$ if $\Gamma$ is either the endsequent of $P$ or the uppersequent of a rule $(s u b)$.

2. $h(\Gamma)=h(\Delta)+1$ if $\Gamma$ is the uppersequent of an $(h g t)$ whose lowersequent is $\Delta$.

3. $h(\Gamma)=h(\Delta)$ if $\Gamma$ is the uppersequent of a rule other than $(s u b)$ and $(h g t)$ and $\Delta$ is the lowersequent.

Again let $P$ be a proof (in $N I D_{q+1}+\forall x B(x)$ ). Let $o$ denote an assignment of an ordinal term $o(\Gamma)=$ $o(\Gamma ; P) \in O T(q+1)$ to each sequent $\Gamma$ in $P$. If the assignment $o: \Gamma \mapsto o(\Gamma)$ enjoys the following conditions, then we say that $o$ is an ordinal assignment for $P$.

1. $o(\Gamma) \neq 0$ for each axiom $\Gamma$.

Assume that $\Gamma$ is the lowersequent of a rule $J$ and $\Gamma_{0}$ and $\Gamma_{1}$ denote the uppersequents of $J$.

2. $o(\Gamma)=o\left(\Gamma_{0}\right)$ if $J$ is one of $(\forall),($ weak $),\left(P_{u}\right)$.

3. $o(\Gamma)=o\left(\Gamma_{0}\right)=o\left(\Gamma_{1}\right)$ if $J$ is $(\Lambda)$.

N.B. We require ordinals assigned to uppersequents of a $(\wedge)$ are equal.

4. $o(\Gamma)=o\left(\Gamma_{0}\right)+b$ for some nonzero $0 \neq b \in O T(q+1)$ if $J$ is either $(\exists)$ or $(\vee)$.

In this case we write, e.g., $(\mathrm{V})_{b}$ for the rule $(\mathrm{V})$.

5. $o(\Gamma)=o\left(\Gamma_{0}\right)+b$ if $J$ is $(p a d)_{b}$.

6. $o(\Gamma)=o\left(\Gamma_{0}\right)+\Omega_{1+u}$ if $J$ is $\left(\neg P_{u}\right)$.

7. $o(\Gamma)=o\left(\Gamma_{0}\right)+o\left(\Gamma_{1}\right)$ if $J$ is $(c u t)$.

8. $o(\Gamma)=D_{q+1} o\left(\Gamma_{0}\right)$ if $J$ is $(h g t)$.

9. $o(\Gamma)=D_{u} o\left(\Gamma_{0}\right)$ if $J$ is $(s u b)_{u}(u \leq q)$.

For an ordinal assignment $o$ for a proof $P$ we set $o(P)=o\left(\Gamma_{\text {end }}\right)$ with the endsequent $\Gamma_{\text {end }}$ of $P$.

\section{Remark.}

1. The padding rule $(p a d)_{b}$ is implicit in the literature, e.g., in [B2].

2. The substitution rule $(s u b)$ comes from [T] but Buchholz mentions a substitution operation $N t \mapsto F t$ in the proof of Lemma 4.12 in [B2].

Let $P$ be a proof in $N I D_{q+1}+\forall x B(x)$ and $o$ an ordinal assignment for $P$. We say that $(P, o)$ is a proof with the o.a. (=ordinal assignment) $o$ if the following conditions are fulfilled:

(p0) The endsequent of $P$ is the empty sequent. 
(p1) The final part of $P$ is an empty $(s u b)_{0}$ followed by a nonempty series $\left\{(p a d)_{b_{i}}\right\}_{i \leq n}$ of paddings with $\operatorname{dom}\left(b_{i}\right) \in\{\emptyset,\{0\}, \omega\}$ :

$$
\begin{gathered}
\frac{\vdots}{\vdots}(s u b)_{0} \\
P \quad(p a d)_{b_{0}} \\
\quad \quad \stackrel{\vdots}{ } \quad(p a d)_{b_{n}}
\end{gathered}
$$

(p2) For any (cut) in $P$,

$$
\frac{\Gamma, \neg A \quad A, \Delta}{\Gamma, \Delta}(c u t)
$$

$|A| \leq h(A, \Delta ; P)=h(\Gamma, \neg A ; P)$.

(p3) For any $\left(\neg P_{u}\right)$ in $P$,

$$
\begin{aligned}
& \frac{\Gamma, t \notin P_{u}, \neg \mathcal{N}_{u}(F, t)}{\Gamma, t \notin P_{u}}\left(\neg P_{u}\right) \\
& \left|\neg \mathcal{N}_{u}(F, t)\right| \leq h\left(\Gamma, t \notin P_{u}, \neg \mathcal{N}_{u}(F, t) ; P\right) .
\end{aligned}
$$

Proposition 4 Assume $I D_{q}+\forall x B(x)$ is inconsistent. Then there exists a proof $P$ with an o.a. o.

Proof. By Lemma 1 pick a proof $P_{0}$ in $N I D_{q+1}$ ending with the empty sequent. $P_{0}$ contains none of rules $(s u b),(p a d),(h g t)$. Below the endsequent of $P_{0}$ attach some $(h g t)$ 's to enjoy the conditions (p2) and (p3). After that attach further a $(s u b)_{0}$ and a $(p a d)_{0}$ to ensure the condition $(\mathbf{p} 1)$. Let $P$ denote the resulting proof in $N I D_{q+1}+\forall x B(x)$ of the empty sequent. For each sequent $\Gamma$ in $P_{0}$ set the ordinal $o(\Gamma)=\Omega_{q+1} \cdot n$ for some $n<\omega$. Then the whole proof $P$ has an ordinal $o(P)=D_{0} D_{q+1}^{k}(\Omega \cdot k)$ for some $k$.

Thus assuming that $\forall x B(x)$ is true, it suffices to show the following lemma.

Lemma 2 Let $(P, o)$ be a proof with an o.a. o. Then there exists a proof $\left(P^{\prime}, o\right)=r(P, o)$ with an o.a. o such that

$$
o\left(P^{\prime}\right)=o(P)[1]
$$

It remains to prove the Lemma 2.

Let $P$ be a proof (not neccessrily ending with the empty sequent). The main branch of $P$ is a series $\left\{\Gamma_{i}\right\}_{i \leq n}$ of sequents in $P$ such that:

1. $\Gamma_{0}$ is the endsequent of $P$.

2. For each $i<n \Gamma_{i+1}$ is the right uppersequent of a rule $J_{i}$ so that $\Gamma_{i}$ is the lowersequent of $J_{i}$ and $J_{i}$ is one of the rules $(c u t),($ weak $),(h g t),(s u b)$ and $(\text { pad })_{0}$.

3. Either $\Gamma_{n}$ is an axiom or $\Gamma_{n}$ is the lowersequent of one of the rules $(\vee),(\exists),\left(\neg P_{u}\right)$ and $(\text { pad })_{b}$ with $b \neq 0$. The sequent $\Gamma_{n}$ is called the top (of the main branch) of the proof $P$.

Let $P$ be a proof with an o.a. and $\Gamma$ a sequent in $P$. The $u$-resolvent of $\Gamma$ is the uppermost substitution rule $(s u b)_{v}$ below $\Gamma$ with $v \leq u$. Note that such a substitution rule always exists by the condition (p 1$)$.

Let $\Phi$ denote the top of the proof $P$ with the o.a. $o$. Put $\alpha=o(P)$. Observe that we can assume $\Phi$ contains no first order free variable.

Case 1. $\Phi$ is the lowersequent of a rule $(p)_{b}$ at which the ordinal $b$ is padded. This means that either $(p)_{b}=(p a d)_{b}$ with $b \neq 0$ or $(p)_{b}=(\vee)_{b},(\exists)_{b}$ with $b>1$.:

$$
\begin{array}{ll} 
& \bar{\Phi}+b,(p)_{b} \\
P & \vdots
\end{array}
$$

Case 1.1. Either the top $\Phi$ is the endsequent (, then the last rule is $(p)_{b}=(p a d)_{b}$ with $\left.b \neq 0\right)$, and/or $\operatorname{dom}(b)=\omega: \operatorname{dom}(\alpha)=\operatorname{dom}(b)$. Replace the rule $(p)_{b}$ by $(p)_{b[1]}$. Note that $b[1] \neq 0$ if $b>1$.

$$
\begin{aligned}
& \bar{\Phi}+b[1],(p)_{b[1]} \\
& P^{\prime} \quad
\end{aligned}
$$


Case 1.2. Otherwise:

Case 1.21. $\operatorname{dom}(b)=T_{u}(q+1)$ : Let $I$ be the $u$-resolvent of $\Phi$ and $\Gamma$ the lowersequent of $J . o(\Gamma)$ is of the form $D_{v} a$ for some $v \leq u$ and $a$ with $\operatorname{dom}(a)=T_{u}(q+1)$. We have $\left(D_{v} a\right)[1]=D_{v} a\left[a_{1}\right]$ with $a_{1}=D_{u} a[1]$. Replace the $(p)_{b}$ by $(p)_{b\left[a_{1}\right]}$.

Case 1.22. $\operatorname{dom}(b)=\{0\}$, i.e., $b=b_{0}+1$ for some $b_{0}$ : Let $J$ denote the uppermost $(s u b)$ or $(h g t)$ below $\Phi$ and $\Gamma$ the lowersequent of $J . o(\Gamma)$ is of the form $D_{v}\left(a+b_{0}+1\right)$ :

$$
\begin{aligned}
& \bar{\Phi}+b_{0}+1 \\
& \vdots \\
& \bar{\Gamma} \\
& \vdots
\end{aligned}
$$

Replace the $(p)_{b_{0}+1}$ by $(p)_{b_{0}}$ and insert a new $(p a d)_{c}$ immediately below $J$ with $c=D_{v}\left(a+b_{0}\right)$ :

$$
\begin{aligned}
& \bar{\Phi}+b_{0} \\
& \vdots \\
& \bar{\Gamma}+D_{v}\left(a+b_{0}\right) J \\
& \frac{\Gamma}{\Gamma}+c \cdot 2(\text { pad })_{c} \\
& \vdots
\end{aligned}
$$

Case 2. $\Phi$ is an axiom and contains a true a.p.f. $A: \Phi=A, \Delta_{0}$.

$$
\begin{aligned}
& A, \Delta_{0} \\
& \text { : } a \quad \vdots b \\
& \frac{\Gamma, \neg A \quad A, \Delta}{\Gamma, \Delta} a+b \\
& P
\end{aligned}
$$

where $a=o(\Gamma, \neg A), b=o(A, \Delta)(b \neq 0)$. Eliminate the false a.p.f. $\neg A$ and insert a $(w e a k)$ and an appropriate $\left(\right.$ pad) as in Case 1 to get $o\left(P^{\prime}\right)=\alpha[1]$.

$$
\begin{gathered}
\vdots a \\
\frac{\Gamma^{\prime}}{\Gamma, \Delta}(\text { weak }) \\
\vdots \quad(\text { pad })
\end{gathered}
$$

Case 3. $\Phi$ is a logical axiom: $\Phi=\neg X(t), X(t), \Delta_{0}$. Put

$X^{+}(t)=X(t), X^{-}(t)=\neg X(t),(\neg X(t))^{-}=X(t),(\neg X(t))^{+}=X(t)$.

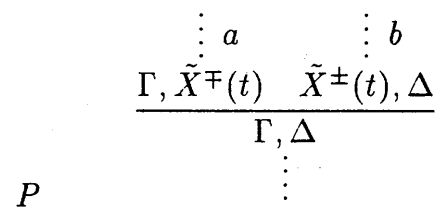

where $\tilde{X}^{\mp}(t) \in \Delta$ and $\tilde{X}$ denotes either $X$ or a formula $F$ by a $(s u b)$. As in Case 2 insert a $($ weak) and a $(p a d)$.

$$
\begin{gathered}
\vdots a \\
\frac{\Gamma, \tilde{X}^{\mp}(t)}{\Gamma, \Delta}(\text { weak }) \\
\vdots(p a d)
\end{gathered}
$$

Case 4. $\Phi$ is the lowersequent of a $(\vee)_{1}$.

Case 5. $\Phi$ is the lowersequent of an $(\exists)_{1}$

Consider the Case 4. Let $J$ denote a (cut) at which the descendent of the principal formula $A_{0} \vee A_{1}$ of the $(\mathrm{V})_{1}$ vanishes.

Case 4.1. There exists an $(h g t)$ or a $(s u b)$ between $\Phi$ and $J$ : Let $I$ denote the uppermost one among such 
rules.

$$
\begin{gathered}
\frac{A_{i}, A_{0} \vee A_{1}, \Delta_{0}}{A_{0} \vee A_{1}, \Delta_{0}}(\vee)_{1}+1 \\
\frac{\vdots}{A_{0}^{\prime} \vee \tilde{A}_{1}^{\prime}, \Delta^{\prime}} I \\
\vdots \\
\frac{\Gamma, \neg \tilde{A}_{0} \wedge \neg \tilde{A}_{1} \quad \tilde{A}_{0} \vee \tilde{A}_{1}, \Delta}{\Gamma, \Delta} J \\
\vdots
\end{gathered}
$$

where 1) $i=0,1,2) \tilde{A}_{i}$ is either $A_{i}$ or $A_{i}[X:=F]$ and 3) $A_{i}^{\prime} \in\left\{A_{i}, \tilde{A}_{i}\right\}$. For the lowersequent of $I$ $o\left(A_{0}^{\prime} \vee A_{1}^{\prime}, \Delta^{\prime}\right)=D_{u}(c+1)$ for some $u \leq q+1$ and a $c$.

Lower the $(\vee)_{1}$ under $I$ and change +1 into $+D_{u} c$ :

$$
\begin{gathered}
A_{i}, A_{0} \vee A_{1}, \Delta_{0} \\
\frac{\vdots}{\frac{A_{i}^{\prime}, A_{0}^{\prime} \vee A_{1}^{\prime}, \Delta^{\prime}}{A_{0}^{\prime} \vee A_{1}^{\prime}, \Delta^{\prime}}} I \\
\vdots \\
\frac{\Gamma, \neg \tilde{A}_{0} \wedge \neg \tilde{A}_{1} c \quad \tilde{A}_{0} \vee \tilde{A}_{1}, \Delta}{\Gamma, \Delta} \\
\vdots
\end{gathered}
$$

Case 4.2. Otherwise: Let $I$ denote the uppermost $(h g t)$ below the $(c u t) J$. Such an (hgt) exists since $\left|\tilde{A}_{0} \vee \tilde{A}_{1}\right|>0$. Put $h=h\left(\tilde{A}_{0} \vee \tilde{A}_{1}, \Delta\right)-1$. Then $h=h(\Lambda) \geq \max \left\{\left|\tilde{A}_{0}\right|,\left|\tilde{A}_{1}\right|\right\}$ for the lowersequent $\Lambda$ of the (hgt) $I$.

$$
\begin{aligned}
& \quad \frac{A_{i}, A_{0} \vee A_{1}, \Delta_{0}}{A_{0} \vee A_{1}, \Delta_{0}}(\vee)_{1}+1
\end{aligned}
$$

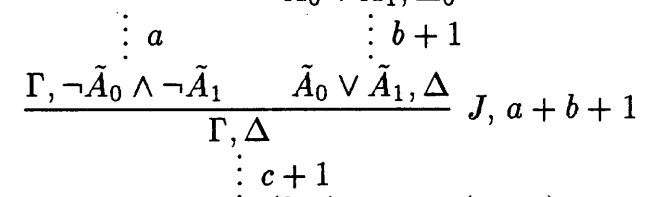

$$
\begin{aligned}
& \frac{\dot{\bar{\Lambda}}}{\grave{ }}(h g t) I, D_{q+1}(c+1)
\end{aligned}
$$

where $a=o\left(\Gamma, \neg \tilde{A}_{0} \wedge \neg \tilde{A}_{1}\right), b+1=o\left(\tilde{A}_{0} \vee \tilde{A}_{1}, \Delta\right)$ and $o(\Lambda)=D_{q+1}(c+1)$ for some $c$.

Assuming $\neg \tilde{A}_{i}$ is an $E$ formula, let $P^{\prime}$ be the following:

$$
\begin{aligned}
& A_{i}, A_{0} \vee A_{1}, \Delta_{0}
\end{aligned}
$$

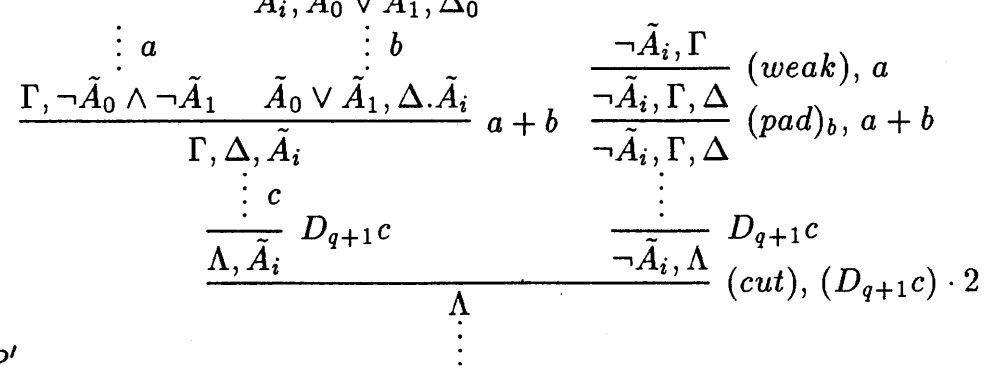

Here the subproof ending with $\neg \tilde{A}_{i}, \Gamma$ is obtained from the subproof of $P$ ending with the left uppersequent $\Gamma, \neg \tilde{A}_{0} \vee \neg \tilde{A}_{1}$ of the $(c u t) J$ by inversion. Observe that we still have $a=o\left(\neg \tilde{A}_{i}, \Gamma ; P^{\prime}\right)$ under the same ordinal assignment since the lowersequent and the uppersequents of a rule $(\wedge)$ have the same assigned ordinal.

Case 6. $\Phi$ is the lowersequent of a $\left(\neg P_{u}\right)$.

Let $J$ denote the (cut) at which the descendent of the principal formula of the $\left(\neg P_{u}\right)$ vanishes and $I$ the $u$-resolvent of $\Phi=t \neg P_{u}, \Delta_{0}$. Here note that there is no $(s u b)_{v}(v \leq u)$ between the $\left(\neg P_{u}\right)$ and $J$ by the restriction: the uppersequent of a $(s u b)_{v} \subseteq P_{o s}$, i.e., $\neg P_{u}(t) \notin P o s_{v}$. Therefore the $u$ resolvent $I$ is below $J$. Also by the definition there is no $(s u b)_{v}(v \leq u)$ between $J$ and $I$. 


$$
\begin{array}{cc} 
& \frac{t \notin P_{u}, \neg \mathcal{N}_{u}(F, t), \Delta_{0}}{t \notin P_{u}, \Delta_{0}}\left(\neg P_{u}\right),+\Omega_{u+1} \\
\vdots & \vdots b \\
\Gamma, t \in P_{u} & t \notin \dot{P}_{u}, \Delta \\
& \Gamma, a+b \\
& \vdots c \\
& \frac{\Lambda}{\tilde{\Lambda}}(s u b)_{u} I, D_{v} c
\end{array}
$$

where $a=o\left(\Gamma, t \in P_{u}\right), b=o\left(t \notin P_{u}, \Delta\right), c=o(\Lambda)$ and $o\left(t \notin P_{u}, \Delta_{0}\right)=b_{0}+\Omega_{u+1}$ with $b_{0}=o(t \notin$ $\left.P_{u}, \neg \mathcal{N}_{u}(F, t), \Delta_{0}\right)$. We have $\operatorname{dom}(b)=\operatorname{dom}(c)=\operatorname{dom}\left(\Omega_{u+1}\right)=T_{u}(q+1)$.

Put $z=D_{u} c[1]$. Let $P^{\prime}$ be the following:

$$
\begin{aligned}
& \vdots a
\end{aligned}
$$

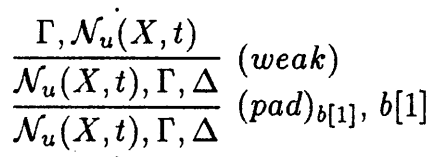

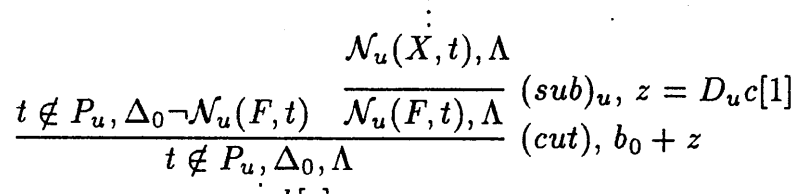

$$
\begin{aligned}
& \vdots a \quad \vdots b[z]
\end{aligned}
$$

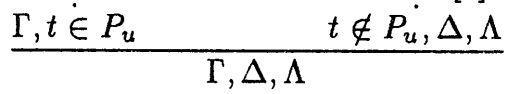

$$
\begin{aligned}
& \vdots c[z] \\
& \frac{\Lambda, \Lambda}{\tilde{\Lambda}} D_{v} c[z]
\end{aligned}
$$

where the subproof ending with $\Gamma, \mathcal{N}_{u}(X, t)$ is obtained from the subproof in $P$ ending with the left uppersequent $\Gamma, t \in P_{u}$ of the $(c u t) J$ by inversion. Note that $\mathcal{N}_{u}(F, t)$ is an $E$ formula. We have $o\left(\Gamma, \mathcal{N}_{u}(X, t) ; P^{\prime}\right)=a=$ $o\left(\Gamma, t \in P_{u} ; P\right)$ and hence $o\left(P_{0}\right)=o\left(\mathcal{N}_{u}(X, t), \Lambda ; P^{\prime}\right)=c[1]$. Thus $o\left(\mathcal{N}_{u}(F, t), \Lambda ; P^{\prime}\right)=D_{u} c[1]=z$ and $o\left(\tilde{\Lambda} ; P^{\prime}\right)=D_{v} c[z]=\left(D_{v} c\right)[1]$ with $D_{v} c=o(\tilde{\Lambda} ; P)$. Therefore $o\left(P^{\prime}\right)=\alpha[1]$.

This completes a proof of the Lemma 2 and hence of the Theorem 1. 
Remark. As in $[\mathrm{A}]$ we have for each $n<\omega$

$$
I \Sigma_{k} \vdash \forall d \exists m\left\{\left(D_{0} D_{1}^{k-1}(\Omega \cdot n)\right)[d]^{m}=0\right\}
$$

From this we can expect to sharpen the Theorem 1 for fragments, e.g., for $I \Sigma_{k}$ but we have no proof of the following:

Show that

$$
I \Sigma_{k} \nvdash \forall n \exists m\left\{\left(D_{0} D_{1}^{k-1}(\Omega \cdot n)\right)[1]^{m}=0\right\}
$$

\section{References}

[A] Arai, T.: A slow growing analogue to Buchholz' proof. Ann. Pure Appl. Logic 54, 101-120 (1991)

[B1] Buchholz, W.: A new system of proof-theoretic ordinal functions. Ann. Pure Appl. Logic 32, 195-207 (1986)

[B2] Buchholz, W.: An independence result for $\left(\Pi_{1}^{1}-C A\right)+B I$. Ann. Pure Appl. Logic 33, 131-155 (1987)

[H-O] Hamano, M., Okada, M.: A direct independence proof of Buchholz's hydra game on finite labeled trees, submitted

[S] Schmerl, U.: Number theory and the Bachmann/Howard ordinal. In: Stern, J (ed.) Proceedings of the Herbrand Symposium Logic Colloquim '81 (Studies in Logic, vol. 107, pp. 287-298). Amsterdam: NorthHolland 1981

[T] Takeuti, G.: Proof Theory. second edition. Studies in Logic. Amsterdam: North-Holland 1987 